\title{
Blockchain Transaction Processing
}

Suyash Gupta, Mohammad Sadoghi

\section{Synonyms}

- Blockchain Data Management

- Blockchain Consensus

- Cryptocurrency

\section{Definitions}

A blockchain is an append-only linked-list of blocks, which is maintained at each participating node. Each block records a set of transactions and their associated metadata. Blockchain transactions act on the identical ledger data stored at each node. Blockchain was first perceived by Satoshi Nakamoto (Satoshi 2008) as a peer-to-peer digital-commodity (also known as crypto-currency) exchange system. Blockchains received traction due to their inherent property of immutability—once a block is accepted, it cannot be reverted.

\section{Overview}

In 2008, Satoshi Nakamoto (Satoshi 2008) introduced the design of an unanticipated technology that revolutionized the research across the distributed systems community. Nakamoto presented the design of a peer-to-peer digital-commodity exchange system, which although employed by several participants, prevents the use of a centralized design. Nakamoto envisioned a system where the participants exchange commodities among themselves in a democratic, decentralized and transparent manner while upholding their right to privacy. Nakamoto visualized this digital-commodity as a monetary token that could be used by participants to provide or receive services. This led to the birth of Bitcoin-a cryptocurrency - and introduction of a new design paradigm Blockchain.

A blockchain in its simplest form is an append-only linked-list of blocks. 


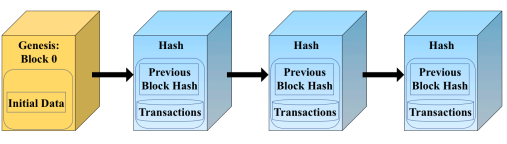

Fig. 1: Basic Blockchain Representations

Each block in this chain is linked to the previous block in the chain Gupta et al (2019a, 2020a). Blockchains are often termed as immutable as modifying an existing block requires modifying all the previous blocks in the chain. Each block includes a set of transactions and the associated meta-data. Figure 1 presents a schematic representation of a blockchain. Blockchain systems guarantee decentralization as the full-copy of the chain is maintained by several participant ${ }^{1}$ Moreover, a block is only accepted into the chain after all the participants have reached consensus on the order and contents of the block. In specific, admittance of a block to the chain implies that the transactions iin the block have been executed and verified. Hence, blockchain helps in achieving key properties such as democracy and transparency.

A blockchain system can be described as a collection of layers. At the application layer, there are clients, which send their transactions to a set of severs to process. The communication among the clients and servers take place at the networking layer. Servers participate at the ordering layer to assign a unique order to each incoming client transaction in a Byzantine FaultTolerant (henceforth referred to as BFT) manner. Following a successful ordering, the client transaction is processed

\footnotetext{
${ }^{1}$ In sharded blockchain systems, no shard may have complete copy but data is still securely replicated.
}

at the execution layer and persisted in the immutable ledger at the storage layer. Clients and servers also employ necessary cryptographic constructs to securely exchange messages among each other.

The preceding discussion allows us to summarize that a blockchain system aims at providing a safe and resilient storage for transactions. In the succeeding sections, we will discuss these concepts in detail and will illustrate the mechanisms pertaining to blockchain transaction processing. We will also study key principles required to order and validate these client transactions and provide analysis of some existing blockchain applications.

\section{Key Research Findings}

Each blockchain system can be visualized as a secure representation of a traditional database system (Nawab 2018). Similar to a database system, each blockchain application also receives transactions from multiple clients. In its vanilla form, a blockchain transaction is a collection of read or write operations. Clients issue these transactions to the servers for processing and exchange of digital-commodities.

In general, each server in a blockchain application stores a fullcopy of the chain. Hence, without any loss of generality, we can claim that servers of a blockchain system are replicas of each other. In specific, a blockchain application lays down a replicated design where each replica participates in ordering and executing the incoming client transaction. 
Prior works have shown that it is possible to make a replicated system handle failures (Lamport 1998, Steen and Tanenbaum 2017). In any replicated system, replicas participate in a faulttolerant consensus protocol to decide the order to execute a client transaction. Blockchain applications also adhere to this philosophy. They employ a BFT consensus protocol to achieve consensus under byzantine failures. But, why do BFT protocols need to handle byzantine failures? As a blockchain system promotes democracy, it permits display of adversarial behavior by malicious replicas during consensus. To tackle such malicious activities, each blockchain application relies on the design and properties dictated by a BFT consensus protocol.

\section{Blockchain Topologies}

A key parameter that renders the design of a blockchain system is its underlying application. On the basis of permissions available to a participating node, a blockchain application can be categorized blockchain as permissioned, permissionless or hybrid (Pilkington 2015. Cachin and Vukolic 2017). Although the blockchain community agrees on the characteristics of a permissionless or public blockchain infrastructure, there is a lack of concise definitions to explain other models.

On the basis of topology, we categorize a blockchain systems under four heads: public, private, permissioned and hybrid. Figure 2 presents a pictorial representation of the different categories. In these figures, nodes that lack any connections are disallowed from participating in the management of the blockchain. Further, we use circles to demarcate different zones of operation; certain nodes are allowed to lead the consensus (or create the next block) while some nodes are allowed to participate in the consensus protocol.

Public Blockchain systems, such as Bitcoin (Satoshi 2008) and Ethereum (Wood 2015), allow any node to participate in the consensus process and propose the next valid block for the chain. Hence, a public or permissionless blockchain system upholds its democratic nature by providing each node with equal probability ${ }^{2}$ of creating the next block to be added to the chain.

Private Blockchain systems run at the other extreme end of the spectrum. These blockchain systems permit only a specific set of nodes to be part of the consensus protocol and restrict the creation of next block to an even smaller subset of nodes. Private blockchain designs are attractive to large multi-sector companies and banks, which may chose to allow some of their customers to participate in the consensus protocol, while restricting creation of next block to its employees.

Hybrid Blockchain systems attain a middle ground between the two extremes. Although these systems allow any node to be part of the consensus protocol, they restrict the task of proposing and creating the next block to a designated subset of replicas. For instance, Ripple (Schwartz et al 2014) - a cryptocurrency-supports a variant of the hybrid model. In Ripple, only some public institutions have the permissions

2 The equal probability of creating a block is only guaranteed when all the nodes have exactly same amount of resources, and each node is working independently. 


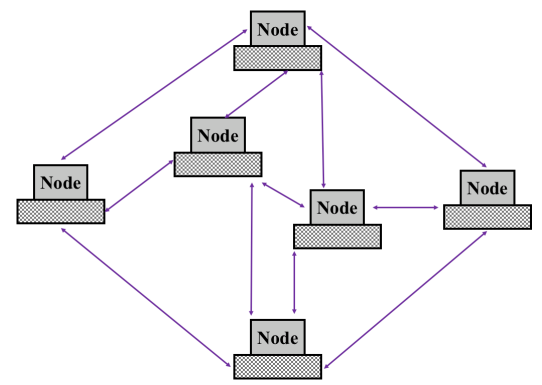

(a) Public Blockchain.

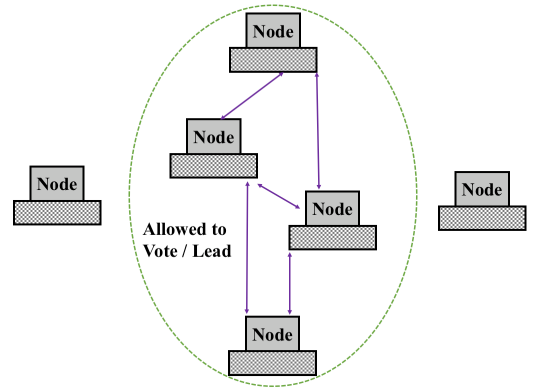

(c) Permissioned Blockchain.

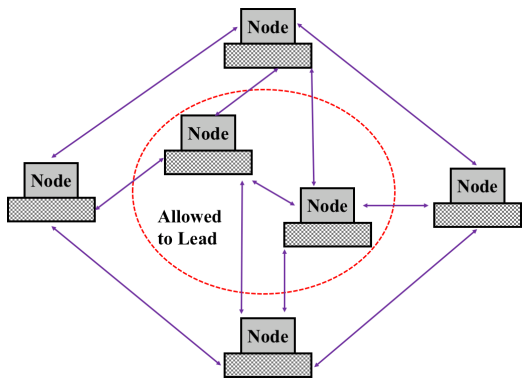

(b) Hybrid Blockchain.

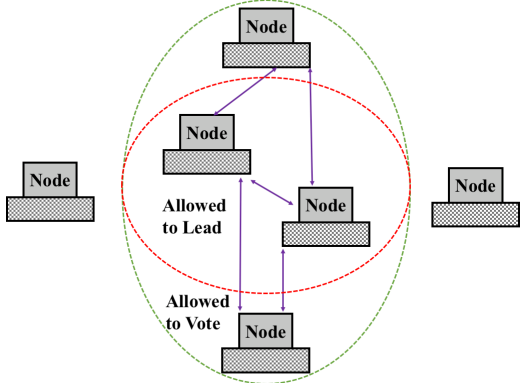

(d) Private Blockchain.

Fig. 2: Topologies for Blockchain Systems.

to select the transactions that will be part of the next block.

Amidst all these topologies, permissioned blockchain systems have successfully created a niche space for their design (Androulaki et al 2018, Gupta et al 2020c). Permissioned blockchain applications allow any node participate in the consensus protocol but require the identities of all participants to be known a priori. Although participants loose their privacy, permissioned blockchain applications provide each participant equal opportunity to propose the next block. Notice that permissioned blockchain applications place no other special restrictions on the behavior of a participant. Hyperledger Fabric (Androulaki et al 2018), Libra coin (Libra 2019) and RESILIENTDB (Gupta et al 2020c) are some of the state-of-the-art permissioned blockchain applications and fabrics.

\section{Blockchain Transactional Flow}

The initial block of any blockchain is termed as the genesis block (Decker and Wattenhofer 2013). Genesis block is a special block that is numbered zero, and is hard-coded in every blockchain application. Each other block links to some previously existing block. Hence, a blockchain grows by appending new blocks to the existing chain.

A transaction in a blockchain system is identical to any distributed or OLTP transaction (TPP Council 2010) that acts 
on some data. Traditional blockchain applications (such as Bitcoin) consist of transactions that represent an exchange of money between two entities (or users). Each valid transaction is recorded in a block, which can can contain multiple transactions, for efficiency. Immutability is achieved by leveraging strong cryptographic properties such as hashing (Katz and Lindell 2007).

Figure 3 illustrates the three main phases required by any blockchain application to create a new block. The client transmits a transactional request to one of the participants. This participating node multicasts the client request to all other nodes. We term this phase as Transaction Dissemination. Once, all the nodes have a copy of client request, they initiate a consensus protocol. The choice of underlying consensus protocol affects the time complexity and resource consumption. The winner of the consensus phase proposes the next block and transmits it to all other nodes. This transmission process is equivalent to adding an entry (block) to the global distributed ledger.

\section{Blockchain Consensus}

At the core of any blockchain application is a BFT consensus protocol which states that given a client transaction, the aim of this consensus protocol is to ensure all the non-faulty replicas assign the same order to this transaction. Depending on the underlying topology, we can broadly categorize consensus protocols into two categories: permissionless consensus protocols and permissioned consensus protocols.
Achieving fault-tolerant distributed consensus is an age-old problem. Commit protocols such as Two-Phase Commit (Gray 1978), Three-Phase Commit (Skeen 1982) and EasyCommit (Gupta and Sadoghi 2018. 2020) help in reaching agreement among the participants in a partitioned distributed databases Qadah and Sadoghi 2018; Qadah et al 2020, Sadoghi and Blanas 2019). However, commit protocols can only handle node failures and are unsafe under message delay or loss.

Paxos (Lamport 1998) and Viewstamped Replication (Oki and Liskov 1988) allow a distributed system of replicas to achieve consensus in the presence of crash-faults. In a system of $n$ replicas, a system employing Paxos for consensus can handle up to $n$ failures where $n \geq 2 f+1$. Notice that these $f$ failures need not be simple replica crashes but can also take form of message losses and delays. However, crash-fault tolerant protocols such as Paxos and Viewstamped Replication cannot handle any malicious behavior.

A byzantine-fault tolerant protocol aims at reaching consensus in a system of $n$ replicas where at most $f$ replicas can act as byzantine and $n \geq 3 f+1$. Traditional BFT protocols promote a primary-backup model where one replica is designated as the primary and other replicas act as backups. It is the task of the primary to initiate consensus among all the backups. Notice that all the above discussed protocols, such as Two-Phase Commit, Paxos and so on, follow the primary-backup model. The key reason primary-backup model is preferred is because of its simplicity and its ability to blame the primary for an unsuccessful consensus. 


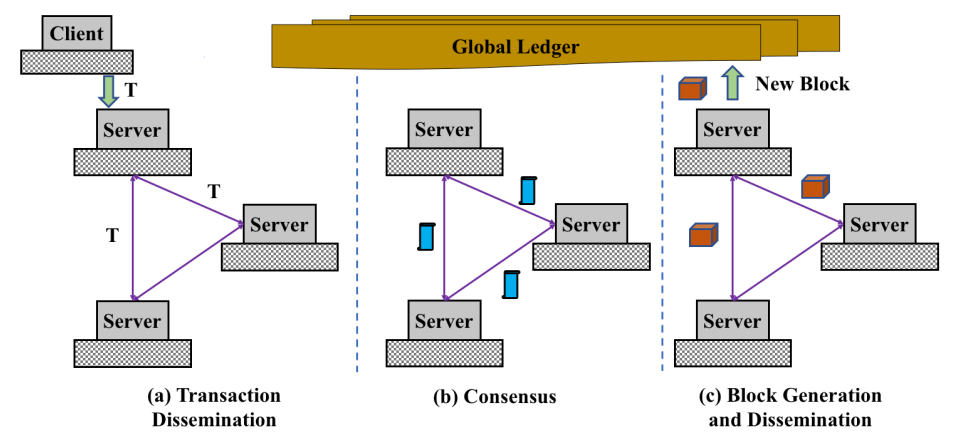

Fig. 3: Blockchain Flow: Three main phases in any blockchain application are represented. (a) Client sends a transaction to one of the server, which it disseminates to all the other servers. (b) Servers run the underlying consensus protocol, to determine the block creator. (c) New block is created, and transmitted to each node, which also implies adding to global ledger.

Recent blockchain applications present several new protocols for achieving consensus: Proof-of-Work (Jakobsson and Juels 1999, Satoshi 2008), Proof-of-Stake (King and Nadal 2012) and Proof-of-Authority (Parity Technologies 2018). Prior works have shown that these consensus protocols provide similar guarantees as traditional BFT protocols (Garay et al 2015). Hence, in the rest of this section, we illustrate some of the state-of-the-art blockchain protocols for both permissioned and permissionless systems.

\section{Permissioned Consensus}

A decade prior to the inception of the first blockchain application, the problem of achieving fault-tolerant distributed consensus problem had already excited practitioners and researchers (Lamport 1998; Oki and Liskov 1988; Castro and Liskov 1999). Distributed systems research community agreed that a byzantine-fault tolerant system can only be considered correct if it is both safe and live. A replicated system is called as safe if all its replicas are consistent, that is, have the same state. A replicated system is termed as live if its replicas are able to make progress, that is, process incoming client requests.

A majority of existing BFT protocols guarantee safety under asynchronous environment, that is, messages can get loss, delayed or duplicated, and up to $f$ replicas may act byzantine. Further, any BFT protocol employs cryptographic constructs to prevent malicious replicas from impersonating non-faulty replicas. As clients send their transactions to other replicas, so each client uses digital signatures to sign its message (Menezes et al 1996, Katz and Lindell 2007). For all other messages, depending on the algorithmic steps, the system can employ either asymmetric-key digital signatures or less-expensive symmetric-key message authentication codes (Katz and Lindell 2007). Hence, we assume authenticated communication: malicious replicas can impersonate each other, but no replica can impersonate a non-faulty replica. Further, replicas will accept only those 
messages which are well-formed, that is, have valid message authentication codes or digital signatures (as applicable).

PBFT. Practical Byzantine Fault Tolerance (Castro and Liskov 1999) if often considered as the first protocol to present a practical design for achieving byzantine fault-tolerance in a distributed system. PBFT follows the primary-backup model where the primary replica initiates the consensus among all the replicas. It is the responsibility of the primary to ensure all the backup replicas successfully order every incoming client transaction otherwise it risks replacement. If the primary is non-malicious and the network is reliable, PBFT guarantees consensus in three phases.

PBFT protocol starts when a client $\mathscr{C}$ wants a transaction to be executed and sends a request $\mathrm{m}$ to the primary replica $\mathscr{P}$. The primary $\mathscr{P}$ checks if the client signature is valid and if this is the case, it creates a PRE-PREPARE message and sends that message to all the backups. This PRE-PREPARE message includes a sequence number (an integer) and a hash of the client request. The sequence number $k$ states the order to execute the transaction while the hash acts as a digest, which can be used in future communications as an alias for the client reques ${ }^{3}$

When a replica $\mathscr{R}$ receives a PRE-PREPARE message from the primary $\mathscr{P}$, it performs the following checks: (i) verifies the client signature on $\mathrm{m}$, (ii) checks if $\mathscr{P}$ is the primary, and (iii) ensures the sequence number $k$ has not already been used. If the PRE-PREPARE message passes all the checks, $\mathscr{R}$ agrees to support primary's order for this request and sends a

\footnotetext{
${ }^{3}$ Client requests are often of the order of several kilobytes and sending an hash instead optimizes the communication.
}

PREPARE message to all the replicas. When a replica $\mathscr{R}$ receives PREPARE messages from $2 f$ replicas in support of the request $\mathrm{m}$ sent by $\mathscr{P}$, then $\mathscr{R}$ marks the request as prepared. This information gives $\mathscr{R}$ an assurance that a majority of non-faulty replicas are also agreeing to order this request at sequence $k$. Next, $\mathscr{R}$ acknowledges the prepared request by sending a COMMIT message to all the replicas. When a replica $\mathscr{R}$ receives COMMIT messages from $2 f+1$ replicas, then $\mathscr{R}$ achieves a unique guarantee on the order of $m$, that is, a majority of non-faulty replicas have also prepared $m$. This allows replica $\mathscr{R}$ to go ahead and execute the request $\mathrm{m}$ as the $k$-th request. Finally, $\mathscr{R}$ sends the result of executing $m$ as a response to the client $\mathscr{C}$.

The client $\mathscr{C}$ needs $f+1$ matching responses from distinct replicas, to mark its request $\mathrm{m}$ as complete. It is possible that the client may not receive sufficient number of matching responses. To handle such cases, the client initiates a timer prior to sending its request. In specific, each client waits on a timer for receiving $f+1$ identical responses. If the client timeouts while waiting for $f+1$ responses, then it forwards its request $\mathrm{m}$ to all the replicas. When a backup replica $\mathscr{R}$ receives a client request $\mathrm{m}$, it forwards that request to the primary $\mathscr{P}$ and starts its timer. If $\mathscr{P}$ fails to send a PRE-PREPARE message corresponding to $\mathrm{m}$, then $\mathscr{R}$ concludes that $\mathscr{P}$ is byzantine and initiates primary replacement. Existing literature terms this primary replacement process as view-change because each primary represents a view of the system. The view-change protocol only starts when at least $f+1$ replicas are ready to replace the primary. This condition is 
necessary as up to $f$ replicas can be byzantine and may even request replacement of a non-faulty primary. Hence, when at least $f+1$ replicas request replacement, remaining replicas assume that there is at least one non-faulty replica which has been affected.

For a successful view-change to take place, a new primary has to be selected. PBFT follows a simple principle: if the replica with index $i$ is the current primary, then replica with index $j$ will be the next primary, where $j=(i+1) \bmod$ $n$. But, how does a replica concludes that it is time for it to act as the new primary. When any replica $\mathscr{R}$ receives VIEW-CHANGE messages from $2 f+1$ distinct replicas that want to elect it as the primary, then it initiates the process of switching to next view. Notice that the process of switching to next view requires ensuring all the replicas have the common state. Thus, the new primary also needs to provide this information as part of the NEW-VIEW message.

Zyzzyva. It is evident from PBFT's design that it requires three phases of communication of which two necessitate quadratic communication complexity. Hence, there is a need for optimized protocols, which can achieve the same goals with much lesser communication overheads. ZyZZYVA (Kotla et al 2007 ) presents a twin-path protocol that achieves consensus in a single linear phase if there are no failures. All the replicas in the ZYZZYVA start in the fast-path and switch to the slow-path under failures. Note that a recent work has illustrated that ZYZZYVA is unsafe under failures (Abraham et al 2017).

In ZYZZYVA, when a non-primary replica $\mathscr{R}$ receives a PRE-PREPARE message from the primary $\mathscr{P}$, it assumes that the primary is non-faulty and agrees to execute this request. Such an execution is termed as speculative as the replica $\mathscr{R}$ is unaware of the state at other replicas. In specific, a byzantine primary could have equivocated and sent different replicas distinct client requests. Once the replica $\mathscr{R}$ executes the request, it sends the reply to client $\mathscr{C}$. The client $\mathscr{C}$ marks the request complete if it receives matching identical responses from at least $3 f+1$ replicas.

A keen reader can easily notice that the onus is on the client to ensure system is safe. Further, when $n=3 f+1$, then the client has to wait for responses from all the replicas. Due to these restrictions, ZYZZYVA's fast-path works only if there are no failures. In ZYZZYVA, the client waits on a timer while expecting $3 f+1$ responses. If the client timeouts prior to receiving responses, then it initiates the slow-path. In the slow-path, client has to summarize the state it received from different replicas and needs to decide whether primary replacement needs to be initiated or a simple recovery protocol is sufficient to ensure system remains live. Clearly, the slow-path is no longer linear and requires multiple phases. Moreover, if the client is malicious, then the replicas could be momentarily unsafe until there is a good client. Another key challenge with twin-path protocols is finding the optimal timeout value. Prior works have shown that finding a timeout value can be hard and ZYZZYVA faces severe reduction is throughput under failures (Clement et al 2009ab; Gupta et al 2021a).

SBFT. The key aim behind the design of SBFT (Golan Gueta et al 2019) is to make a consensus protocol that can guarantee safe consensus with linear message complexity in periods of no 
failures. In fact, like ZYZZYVA, SBFT is also a twin-path protocol. SBFT employs threshold signatures to achieve linear communication complexity.

Threshold signatures are based on asymmetric cryptography. In specific, each replica holds a distinct private key, which it can use to create a signature share. Next, one can produce a valid threshold signature given at least $t$ such signature shares from distinct replicas (the exact value of $t$ is dependent on the underlying consensus protocol).

At a closer look, it seems like SBFT requires more phases than PBFT. This occurs because SBFT linearizes each phase of PBFT through use of threshold signatures. In SBFT, when a replica $\mathscr{R}$ receives a PRE-PREPARE message, it agrees to support from the primary's sequence by generating a threshold share. The replica $\mathscr{R}$ sends this share to a specific replica designated as the collector. When a collector receives message from at least $3 f+2 c+1$ replicas it generates a threshold signatures and sends this signature to all the replicas. When a replica receives a threshold signature from the collector, it executes the request to generate a response, creates a threshold share on this response and sends these to a specific replica designated as the executor. The executor waits for $f+1$ identical responses and combines them into threshold signature. Next, the executor sends this signature to all the replicas and clients.

For SBFT's fast path to work as stated, either there should be no failures or at least $3 f+2 c+1$ replicas should participate in consensus where up to $c>0$ replicas can crash-fail (no byzantine failures). Moreover, the primary can act as both collector and executor but SBFT suggests using distinct replicas in fast path. If the collector timeouts waiting for threshold shares from $3 f+c+1$ replicas, it switches to the slow path, which requires two additional linear phases to complete consensus.

HotStuff. In any primary-backup BFT protocol, if the primary acts malicious, then the protocols employ the accompanying view-change algorithm to detect and replace the malicious primary. This view-change algorithm leads to a momentary disruption in system throughput until the resumption of service.

HotstuFf (Yin et al 2019) proposes eliminating the dependence of a BFT consensus protocol from one primary by replacing primary at the end of every consensus. Although this rotating leader design escapes the cost of a view-change protocol, it enforces an implicit sequential paradigm. Each primary needs to wait for its turn before it can propose a new request.

In HotstufF, in round $i$, the replica with identifier $i \bmod n$ acts as the primary and proposes a request to all the replicas. Each replica on receiving this request, creates a threshold share and sends to the replica $\mathscr{R}$ with identifier $(i+1) \bmod n$. If $\mathscr{R}$ receives threshold shares from $2 f+1$ replicas, then it combines them into a threshold signature and initiates the consensus for round $i+1$ by broadcasting its proposal along with the computed threshold signature. Notice that replicas have not executed the request and replied to the client. HoTSTUFF's aim is to linearize the consensus proposed by PBFT protocol, which it does by splitting each phase of PBFT into two using threshold signatures. To reduce the communication, it chains the phases. Hence, a replica executes the request for the $i$-th round once it receives 
a threshold signature from the primary of $(i+3)$-th round. Evidently, chaining helps HotsTUFF to some extent but it does not eliminate its sequential nature. This sequential nature forces HoTSTUFF to loose out on an opportunity to process messages out-of-order.

PoE. Proof-of-Execution (henceforth referred to as POE) consensus protocol aims at achieving consensus in three linear phases without relying on any twin-path model (Gupta et al 2021a). Further, PoE recognizes that no one size fits all systems (Singh et al 2008). Hence, its design is independent of the choice of underlying cryptographic signature scheme. This implies that the PoE protocol can employ both symmetric and asymmetric-cryptographic signature schemes depending on the application environment.

The design of PoE is built on three key insights. First, PoE prevents use of any twin-path paradigm as switching from fast to slow-path requires dependence on timeouts, which degrades system performance. Second, PoE allows replicas to speculatively execute the requests but facilitates rollbacks in case of inconsistencies. Final, PoE allows out-of-order processing, which eliminates any bottlenecks associated with sequential consensus protocols.

For the sake of brevity, we will describe PoE built on top of threshold signatures. In PoE, the client $\mathscr{C}$ initiates execution by sending its request $\mathrm{m}$ to the primary $\mathscr{P}$. To initiate replication and execution of $\mathrm{m}$ as the $k$-th transaction, the primary proposes $\mathrm{m}$ to all replicas by broadcasting a PROPOSE message.

After a replica $\mathscr{R}$ receives a PROPOSE message from $\mathscr{P}$, it checks whether at least $2 f$ other replicas also received the same proposal from $\mathscr{P}$. To perform this check, each replica agrees to support the first $k$-th proposal it receives from the primary by sending a SUPPORT message that includes its unique threshold share to the primary. The primary $\mathscr{P}$ waits for $2 f+1$ threshold shares, and on receiving such shares, it combines them into a threshold signature and broadcasts as a CeRTIFY message. When a replica $\mathscr{R}$ receives the CERTIFY message, it view-commits to $\mathrm{m}$ as the $k$-th transaction in view $v$. After $\mathscr{R}$ view-commits to $\mathrm{m}$, $\mathscr{R}$ schedules speculative execution of $\mathrm{m}$. Consequently, $\mathrm{m}$ will be executed by $\mathscr{R}$ after all preceding transactions are executed. After execution, $\mathscr{R}$ informs the client of the order of execution and of any execution result. A client considers its transaction successfully executed after it receives identical response messages from $2 f+1$ distinct replicas.

Aardvark. The design philosophy behind Aardvark is distinct in comparison to existing BFT protocols (Clement et al 2009b). It aims at building a robust BFT protocol that can continue performing under failures. Hence, in the failure-free cases, Aardvark attains lower throughput than a majority of the existing BFT protocols.

In Aardvark, prior to sending its request to the primary, the client signs the request using both digital signatures and message authentication codes. This prevents malicious clients from performing a denial-of-service attack as it is expensive for client to sign each message twice. Aardvark also employs a point-to-point network rather than the multicast network for exchange of messages among clients and replicas. The key intuition behind such a choice is to disallow a faulty client or replica from blocking the complete network. 
Aardvark also periodically changes the primary replica. Each replica tracks the throughput of the current primary and suggests replacing the primary when there is a decrease in its throughput. To perform such tracking, each replica sets a timer and measures the rate of primary's responses.

RBFT. The key intuition behind the design of RBFT is to facilitate detection of clever malicious primaries (Aublin et al 2013). RBFT extends Aardvark and aims to detect those malicious primaries, which cannot be detected by simple timers suggested by Aardvark.

In Aardvark, a clever primary can avoid detection by delaying messages just slightly below the timeout threshold. Such a primary can throttle the system throughput without risking eviction. To tackle this challenge, RBFT insists running $f+1$ independent instances of the Aardvark protocol on each replica. One of these instances is designated as the master while other instances act as backups. Irrespective of the designation of an instance, all the instances order all the requests. However, only the master instance executes the requests.

The key task of the backup instances is to monitor the performance of the master instance. If any backup instance observes a degradation of the system throughput at the master, it broadcasts a message to elect a new primary. Further, to guarantee at least one of the $f+1$ instances is led by a non-faulty replica, RBFT requires each instance to be led by a distinct replica. In comparison to both PBFT and Aardvark, RBFT requires an additional phase, which is used to propagate the client requests across all the replicas.

RCC. Although RBFT successfully utilizes redundancy to detect clever malicious primaries, it also wastes excessive bandwidth by requiring all the instances to order the same set of requests. Resilient Concurrent Consensus (henceforth referred to as RCC) paradigm resolves this issue by parallelizing the consensus (Gupta et al 2019b 2021b). In specific, RCC runs at each replica multiple instances of a primary-backup protocol.

The key challenge with the design of primary-backup protocols is their reliance on the primary. This dependence can severely affect the throughput and scalability of these protocols. The primary replica not only receives all client requests but is also responsible for ensuring consensus is reached on the order for these requests among all other replicas. If the primary fails to ensure consensus, then all remaining replicas need to replace this primary. This replacement process is necessary as, without it, nonfaulty replicas may never converge. Unfortunately, primary replacement is not cheap, as it requires pausing consensus on all outstanding requests until the primary is replaced.

RCC aims at making a BFT consensus primary agnostic. To achieve such a property, RCC advocates running $z$ parallel instances at each replica. Further, RCC ensures that each instance is managed by a distinct replica. Using parallelization, RCC ensures that the nonfaulty replicas are always accepting and ordering client requests, this independent of any malicious behavior or attack.

We now present the design of RCC paradigm that parallelizes the seminal PBFT consensus protocol. For the sake of explanation, we assume RCC works in rounds. Each round of RCC includes three stages: parallel consensus, unification, and execution. The notion of a 
round helps in generating a common order and recovering from instance failures but it does not prevent individual primaries from working independently.

Prior to any round, $\mathrm{RCC}$ requires each replica to prepare to run $z$ instances of PBFT protocol in parallel. A round $r$ begins when the primary of each instance proposes a client request. Firstly, in the parallel consensus stage, each instance runs PBFT on its client request. Secondly, in the unification stage, the replica waits for all its $z$ instances to complete replication (reach consensus on their respective requests). If every instance successfully replicates a request, then a common order for execution of these requests is determined. If one or more instances are unable to replicate requests, then the primaries for those instances must be faulty and recovery is initiated. Finally, in the execution stage, each replica executes all the client requests in the common order.

GeoBFT. Existing BFT protocols do not distinguish between the local and global communication, which is a necessary requirement to enable geo-scale deployment of a blockchain system. To resolve this challenge, Geo-Scale Byzantine Fault-Tolerant consensus protocol (henceforth referred to as GEOBFT) that uses topological information to group all replicas in a single region into a single cluster (Gupta et al 2020b). Likewise, GEOBFT assigns each client to a single cluster. This clustering helps in attaining high throughput and scalability in geoscale deployments. GEOBFT operates in rounds, and in each round, every cluster will be able to propose a single client request for execution. Each round consists of the three steps: local replication, global sharing, and ordering and execution, which we further detail next.
At the start of each round, each cluster chooses a single transaction of a local client. Next, each cluster locally replicates its chosen transaction in a Byzantine fault-tolerant manner using PBFT. At the end of successful local replication, PBFT guarantees that each non-faulty replica can prove successful local replication via a commit certificate.

Next, each cluster shares the locallyreplicated transaction along with its commit certificate with all other clusters. To minimize inter-cluster communication, we use a novel optimistic global sharing protocol. Our optimistic global sharing protocol has a global phase in which clusters exchange locally-replicated transactions, followed by a local phase in which clusters distribute any received transactions locally among all local replicas. Finally, after receiving all transactions that are locally-replicated in other clusters, each replica in each cluster can deterministically order all these transactions and proceed with their execution. After execution, the replicas in each cluster inform only local clients of the outcome of the execution of their transactions (e.g., confirm execution or return any execution results).

\section{Permissionless Consensus}

Permissionless applications inspired by Nakamoto's Bitcoin (Satoshi 2008) advocate a public blockchain system where any replica can participate in the consensus. Hence, the identity of a participating replica can be protected. This design property requires the underlying consensus protocol used to order the transactions to expend the resources of a partic- 
ipant. In specific, each participant needs to spend some of its resources if it wants to propose the next block. If such a resource consumption is not enforced, then a malicious participant can create multiple pseudonymous identities and subvert the system, also known as the Sybil attack (Douceur 2002).

Proof-of-Work. Bitcoin relies on the Proof-of-Work (henceforth referred to as PoW) protocol to achieve consensus among a set of replica. PoW protocol builds on top of a simple intuition "What is mathematically hard to compute but easy to verify?" Hence, PoW protocol requires the computation to be expensive, that is, it should deplete some resources of the prover.

In PoW protocol, the participating nodes compete among themselves to propose the next block by solving a complex puzzle. In nature, several computationally hard problems exist, such as Diophantine Equation, RSA Factorization, One-way Hash Functions, and so on. Among these hard problems, following the Nakamoto's vision, PoW protocol is associated with the computation of one-way hash functions such as computation of a 256-bit SHA3 value. When a node $N$ successfully computes this hash value, it disseminates this solution to all other nodes for verification. Any node can verify this solution to check $N$ 's claim.

The main critic behind PoW's design is that leads to excessive wastage of energy. Permissionless applications that employ PoW consensus have to set large targets to prevent Sybil attacks. Further, PoW's design facilitates unfair practices-higher the computational capabilities a node has higher are its chances of solving the complex puzzle. Such a design promotes pooling of resources where several nodes work together to compute the hash. Moreover, a node has to be given incentives to participate in the PoW consensus. If the incentives are not sufficient, then nodes may decline creating the next block, which in turn can either stall the system or compromise its security.

Another issue with the PoW consensus protocol is that it can lead to tricky situations where it is hard to determine the next block in the chain. For instance, two nodes $N_{1}$ and $N_{2}$ may solve the complex puzzle at the same time. In such a case, it is possible that one half of the remaining participants may receive a solution from $N_{1}$ before $N_{2}$ while the other half receives solution from $N_{2}$ before $N_{1}$. To handle this scenario, some form of resolution mechanism is needed, which would lead to wastage of resources of either $N_{1}$ or $N_{2}$ as both of their blocks cannot be appended to the chain. Notice that any new block added to the chain includes the hash of the previous block.

Proof-of-Stake. In PoW protocol, miners have to deplete their computational resources in order to earn the right to create the next block. Each miner who controls a fraction $s$ of the total computational power, has a probability nearly equal to $s$ to create the next block.

Proof-of-Stake (henceforth referred to as POS) presents a principle that contrasts the resource usage philosophy of PoW. In a blockchain system employing PoS protocol, a replica possessing a higher stake than the other replicas gets a chance to create a new block (Bentov et al 2016). In specific, the probability a replica possessing a fraction $s$ of the total stakes in the system creates the next block is $s$. The key security rationale behind PoS is that replicas who have some stake involved 
in the system are also well-suited to ensure its security.

PPCoin or PeerCoin (King and Nadal 2012) is often regarded as the first implementation of PoS. The key motivation behind PPCoin's design was to implement a crypto-currency that does not require participating replicas to spend its resources in performing large computations. Initial PoS-based design were based on the notion of coinage. In specific, a replica's ability to create the next block is determined on its value of coinage. Coinage is calculated on the basis of number of days a replica has held some coins or stake. To prevent Sybil attacks, PoS-based systems require replicas to algorithm requires a node to spend its coinage if it wants to propose the next block.

Initial implementations of the POS protocol lacked the fairness criterion. This is evident as the replica with the highest stake gets the chance to propose the next block. Although a high stake replica looses its coinage once it creates the next block, it may create the subsequent block if its stake is much larger in value than that of the other replicas.

To resolve this issue, a chain-based variant of PoS algorithm has been proposed. The chain-based PoS protocol employs a psuedo-random algorithm to select a validator, which then creates a new block and adds it to the existing chain of blocks. The frequency of selecting the validator is set to some pre-defined time interval. Another variant of PoS algorithm follows BFT-style consensus. In this design, the replicas participate in a BFT protocol to select the next valid block. Here, validators are given right to propose the next block, at random. The key difference between these algorithms is the synchrony re- quirement; chain-based PoS algorithms are inherently synchronous, while BFT-style POS is partially synchronous.

Another key challenge for PoS-based designs is an attack by rational stakeholders. A rational replica would always aim at maximizing its profit, an expected behavior in a democracy in correspondence with the Nash equilibrium Bentov et al (2016). Rational replicas can affect the security of PoS, as in at attempt to maximize their gains, they may participate in multiple chains.

A rational miner could get blocks from distinct forks of the blockchain. To maximize its returns, a miner would attempt to propose the next block for each such fork. As miners don't lose any actual resources (like computational energy in PoW), so they are free to propose blocks on different chains. This could lead to an ever-expanding divergent network.

Proof-of-Authority. A variation of PoS algorithm to be employed in hybrid blockchain topologies is termed as Proof-of-Authority (henceforth referred as PoA) (Parity Technologies 2018). The key idea is to designate a set of nodes as the authorities or leaders. These authorities are entrusted with the task of creating new blocks and validating the transactions. PoA marks a block as part of the blockchain if it is signed by majority of the authorized nodes. The incentive model in POA highlights that it is in the interest of an authority node to maintain its reputation. In case an authority acts malicious, it can loose its status and periodic incentives. Hence, PoA does not select nodes based on their claimed stakes.

Proof-of-Space. A consensus algorithm orthogonal to the design proposed by PoW is proof-of-space 
or proof-of-capacity (henceforth referred as PoC) (Ateniese et al 2014; Dziembowski et al 2015).

PoC expects nodes to provide a proof that they have sufficient "storage" to solve a computational problem. PoC algorithm targets computational problems such as hard-to-pebble graphs (Dziembowski et al 2015) that need large amount of memory storage to solve the problem. In the PoC algorithm, the verifier first expects a prover to commit to a labeling of the graph, and then it queries the prover for random locations in the committed graph. The key intuition behind this approach is that unless the prover has sufficient storage, it would not pass the verification. SpaceMint (Park et al 2015) - a PoC-based cryptocurrency-claims that PoC based approaches are more resource efficient in comparison to $\mathrm{POW}$ as storage consumes less energy.

\section{Blockchain Systems}

We now briefly look at the design of some of the state-of-the-art blockchain applications and fabrics. The key aim of this section is to illustrate the different design practices adopted by existing blockchain systems.

Bitcoin (Satoshi 2008) is regarded as the first ever blockchain application. It is a cryptographically secure digital currency designed with the aim of disrupting the traditional institutionalized monetary exchange. Bitcoin acts as the token of transfer between two parties undergoing a monetary transaction. The underlying blockchain system is a network of nodes (also known as miners) that take a set of client transactions and validate the same by demonstrating a proof-of-work, that is generating a block. The process of generating the next block is non-trivial and requires large computational resources. Hence, the miners are given incentives (such as Bitcoins) for dedicating their resources and generating the block. Each miner maintains locally an updated copy of the complete blockchain and the associated ledgers for every Bitcoin user.

To ensure Bitcoin system remains fair towards all the machines, the difficulty of proof-of-work challenge is periodically increased. Prior works have illustrated that Bitcoin is vulnerable to $51 \%$ attack, which can lead to double spending (Rosenfeld 2014). The intensity of such attacks increases when multiple forks of the longest chain are created. To avoid these attacks, Bitcoin developers suggest the clients to wait for their block to be confirmed before they mark the Bitcoins as transferred. This wait ensures that the specific block is a little deep (nearly six blocks) in the longest chain (Rosenfeld 2014). Bitcoin critics also argue that its proof-of-work consumes huge energy ${ }^{4}$ and may not be a viable solution for future.

Ethereum (Wood 2015) is blockchain framework that permits users to create their own applications (smart-contracts) on top of the Ethereum Virtual Machine (EVM). Ethereum utilizes the notion of smart contracts to facilitate development of new operations. It also supports a digital cryptocurrency, ether, which is used to incentivize the developers to create correct applications. One of the key advantage of Ethereum is that it

\footnotetext{
4 As per some claims one Bitcoin transaction consumes power equivalent to that required by 1.5 American homes per day.
} 
supports a Turing complete language to generate new applications on top of EVM. At the time of writing this article, Ethereum employs a variant of PoW protocol to achieve consensus among its miners. Ethereum makes its miners solve challenges that were not only computational intensive, but also memory intensive. This design prevented existence of miners who utilized specially designed hardware for compute intensive applications.

In future, Ethereum Foundation aims to switch to a variant of PoS protocol to reach consensus among its replicas. The modified protocol is referred to as Casper (Buterin and Griffith 2017). Casper introduces the notion of finality, that is, it ensures that one chain becomes permanent in time. It also introduces the notion of accountability, which penalizes any validator that attempts the nothing-at-stake attack. The penalty leveraged on such a validator is equivalent to negating all his stakes.

Parity (Parity Technologies 2018) is an application designed on top of Ethereum. It provides an interface for its users to interact with the Ethereum blockchain. Parity allows its blockchain community to use either Proof-of-Work and Proof-of-Authority to reach consensus in their applications. Hence, if some users select POA consensus, then Parity provides mechanisms for setting up the authority nodes.

Ripple (Schwartz et al 2014) is considered as third largest cryptocurrency after Bitcoin and Ethereum in terms of market cap. It employs a consensus algorithm which is a simple variant of existing traditional BFT algorithms. Ripple requires number of failures $f$ to be bounded as follows: $\leq(n-1) / 5+1$, where $n$ represents the total number of nodes. Ripple's consensus algorithm introduces the notion of a Unified Node List (UNL), which is a subset of the network. Each server communicates with the nodes in its UNL for reaching a consensus. The servers exchange the set of transactions they received from the clients and propose those transactions to their respective UNL for vote. If a transaction receives $80 \%$ of the votes, it is marked permanent. Notice that if the generated UNL groups are a clique then forks of the longest chain could co-exist. Hence, UNLs are created in a manner that they share some set of nodes. Another noteworthy observation about Ripple protocol is that each client needs to select a set of validators or unique nodes that they trust. These validators utilize the ripple consensus algorithm to verify the transactions.

Hyperledger (Cachin 2016) is a suite of resources aimed at modeling industry standard blockchain applications. It provides a series of Application Programming Interfaces (APIs) for developers to create their own non-public blockchain applications. Hyperledger provides implementations of blockchain systems that uses RBFT and other variants of the PBFT consensus algorithm. It also facilitates use and development of smart contracts. It is important to understand that the design philosophy of Hyperledger leans towards blockchain applications that require existence of non-public networks, and so, they do not need a compute intensive consensus.

ResilientDB (Gupta et al 2020c; Rahnama et al 2020) is a state-of-the-art permissioned blockchain fabric, which is designed with the aim of fostering academic and industry research. RESILIENTDB also acts as a reliable test-bed to implement and evaluate 


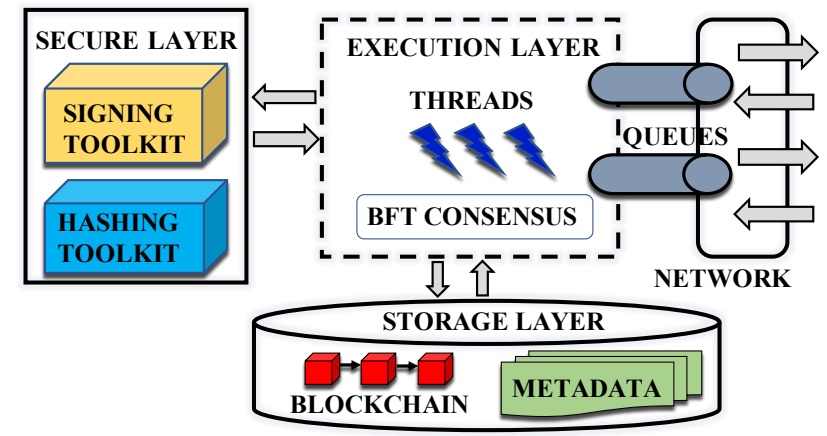

Fig. 4: Architecture of ResilientDB.

enterprise-grade blockchain applications 5 RESILIENTDB evolved from the ExpoDB platform (Sadoghi 2017; Gupta and Sadoghi 2018) which is an experimental research platform to design and test emerging database technologies, agreement and concurrency control protocols.

In Figure 4, we illustrate the overall architecture of RESILIENTDB, which lays down an efficient client-server architecture. At the application layer, we allow multiple clients to co-exist, each of which creates its own requests. For this purpose, they can either employ an existing benchmark suite or design a Smart Contract suiting to the active application. Next, clients and replicas use the transport layer to exchange messages across the network. RESILIENTDB also provides a storage layer where all the metadata corresponding to a request and the blockchain is stored. At each replica, there is an execution layer where the underlying consensus protocol is run on the client request, and the request is ordered and executed. During ordering,

5 RESILIENTDB is open-sourced at https://resilientdb.com and code is available at https://github.com/resilientdb.

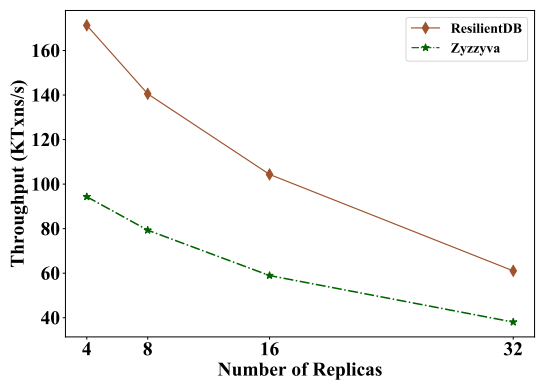

Fig. 5: Two permissioned applications employing distinct BFT protocols $(80 \mathrm{~K}$ clients per experiment).

the secure layer provides support for cryptographic constructs.

RESILIENTDB is written entirely in $\mathrm{C}++$ and provides a graphical user interface to ease user interaction with the system. Further, it also provide a Dockerized deployment that allows any user to experience and test the RESILIENTDB fabric (comprising of multiple replicas and clients) on its local machine.

The key motivation behind RESILIENTDB's design was to show that a system-centric permissioned blockchain fabric can outperform a protocol-centric blockchain fabric even if the former is made to employ a slow consensus 
protocol. To prove this claim, we refer to Figure 5, which compares the throughput achieved by two permissioned fabrics. In this figure, RESILIENTDB employs the slow PBFT protocol while the other fabric adopts the practices suggested in the paper BFTSmart (Bessani] et al 2014) and employs the single-phase linear ZYZZYVA protocol. Despite this disadvantageous choice of consensus protocol, RESILIENTDB achieves a throughput of $175 \mathrm{~K}$ transactions per second, scales up to 32 replicas, and attains up to $79 \%$ more throughput.

\section{Future Directions for Research}

Although blockchain technology is just a decade old, it gained majority of its momentum in the last five years. This allows us to render different elements of the blockchain systems and achieve higher performance and throughput. Some of the plausible directions to develop efficient blockchain systems are: (i) reducing the communication messages, (ii) defining efficient block structure, (iii) improving the consensus algorithm, and (iv) designing secure light-weight cryptographic functions

Statistical and machine learning approaches have presented interesting solutions to automate key processes such as Face Recognition (Zhao et al 2003), Image classification (Krizhevsky et al 2012), Speech Recognition (Graves et al 2013) and so on. The tools can be leveraged to facilitate easy and efficient consensus. The intuition behind this approach is to allow learning algorithms to select nodes, which are fit to act as a block creator and prune the rest from the list of possible creators. The key observation behind such a design is that the nodes selected by the algorithm are predicted to be non-malicious. Machine learning techniques can play an important role in eliminating the human bias and inexperience. To learn which nodes can act as block creators, a feature set, representative of the nodes, needs to be defined. Some interesting features can be: geographical distance, cost of communication, available computational resources, available memory storage and so on. These features would help in generating the dataset that would help to train and test the underlying machine learning model. This model would be ran against new nodes that wish to join the associated blockchain application.

The programming languages and software engineering communities have developed several works that provide semantic guarantees to a language or an application (Wilcox et al 2015; Leroy 2009, Kumar et al 2014). These works have tried to formally verify (Keller 1976; Leroy 2009) the system using the principles of programming languages and techniques such as finite state automata, temporal logic and model checking (Grumberg and Long 1994; Baier and Katoen 2008). We believe similar analysis can be performed in the context of blockchain applications. Theorem provers (such as Z3 (De Moura and Bjørner 2008) and proof assistants (such as COQ (Bertot 2006) could prove useful to define a certified blockchain application. A certified blockchain application can help in stating theoretical bounds on the resources required to generate a block. Similarly, some of the blockchain consensus has been shown to suffer from Denial of Service attacks (Bonneau et al 2015), and a formally verified blockchain application 
can help realize such guarantees, if the underlying application provides such a claim.

\section{References}

Abraham I, Gueta G, Malkhi D, Alvisi L, Kotla R, Martin J (2017) Revisiting fast practical byzantine fault tolerance. URL https: / / arxiv.org/abs/1712.01367

Androulaki E, Barger A, Bortnikov V, Cachin C, Christidis K, Caro AD, Enyeart D, Ferris C, Laventman G, Manevich Y, Muralidharan S, Murthy C, Nguyen B, Sethi M, Singh G, Smith K, Sorniotti A, Stathakopoulou C, Vukolic M, Cocco SW, Yellick J (2018) Hyperledger fabric: A distributed operating system for permissioned blockchains. CoRR abs/1801.10228, URL http://arxiv. org/abs/1801.10228

Ateniese G, Bonacina I, Faonio A, Galesi N (2014) Proofs of space: When space is of the essence. In: Abdalla M, De Prisco R (eds) Security and Cryptography for Networks, Springer International Publishing, pp 538557

Aublin PL, Mokhtar SB, Quéma V (2013) RBFT: Redundant Byzantine Fault Tolerance. In: Proceedings of the 2013 IEEE 33rd International Conference on Distributed Computing Systems, IEEE Computer Society, ICDCS '13, pp 297-306

Baier C, Katoen JP (2008) Principles of Model Checking (Representation and Mind Series) The MIT Press

Bentov I, Gabizon A, Mizrahi A (2016) Cryptocurrencies without proof of work. In: Clark J, Meiklejohn S, Ryan PY, Wallach D, Brenner M, Rohloff K (eds) Financial Cryptography and Data Security, Springer Berlin Heidelberg, Berlin, Heidelberg, pp 142-157

Bertot Y (2006) Coq in a Hurry. CoRR abs/cs/0603118, URL http://arxiv. org/abs/cs/0603118. cs/0603118

Bessani A, Sousa J, Alchieri EEP (2014) State machine replication for the masses with bftsmart. In: DSN

Bonneau J, Miller A, Clark J, Narayanan A, Kroll JA, Felten EW (2015) SoK: Research Perspectives and Challenges for Bitcoin and
Cryptocurrencies. In: Proceedings of the 2015 IEEE Symposium on Security and Privacy, IEEE Computer Society, Washington, DC, USA, SP '15, pp 104-121

Buterin V, Griffith V (2017) Casper the Friendly Finality Gadget. CoRR abs/1710.09437, URL http://arxiv.org/abs/ 1710.09437 . 1710.09437

Cachin C (2016) Architecture of the Hyperledger blockchain fabric. In: Workshop on Distributed Cryptocurrencies and Consensus Ledgers, DCCL 2016

Cachin C, Vukolic M (2017) Blockchain Consensus Protocols in the Wild. CoRR abs/1707.01873

Castro M, Liskov B (1999) Practical byzantine fault tolerance. In: Proceedings of the Third Symposium on Operating Systems Design and Implementation, USENIX Association, Berkeley, CA, USA, OSDI '99, pp 173-186

Clement A, Kapritsos M, Lee S, Wang Y, Alvisi L, Dahlin M, Riche T (2009a) Upright cluster services. In: Proceedings of the ACM SIGOPS 22nd Symposium on Operating Systems Principles, ACM, SOSP, pp 277290, DOI 10.1145/1629575.1629602

Clement A, Wong E, Alvisi L, Dahlin M, Marchetti M (2009b) Making byzantine fault tolerant systems tolerate byzantine faults. In: Proceedings of the 6th USENIX Symposium on Networked Systems Design and Implementation, USENIX Association, NSDI, pp 153-168

De Moura L, Bjørner N (2008) Z3: An Efficient SMT Solver, Springer Berlin Heidelberg, Berlin, Heidelberg, pp 337-340

Decker C, Wattenhofer R (2013) Information Propagation in the Bitcoin Network. In: 13th IEEE International Conference on Peer-toPeer Computing (P2P), Trento, Italy

Douceur JJ (2002) The sybil attack. In: Proceedings of 1st International Workshop on Peer-to-Peer Systems (IPTPS)

Dziembowski S, Faust S, Kolmogorov V, Pietrzak K (2015) Proofs of space. In: Advances in Cryptology - CRYPTO 2015, Springer Berlin Heidelberg, pp 585-605

Garay J, Kiayias A, Leonardos N (2015) The Bitcoin Backbone Protocol: Analysis and Applications, Springer Berlin Heidelberg, Berlin, Heidelberg, pp 281-310

Golan Gueta G, Abraham I, Grossman S, Malkhi D, Pinkas B, Reiter M, Seredinschi D, Tamir O, Tomescu A (2019) Sbft: 
A scalable and decentralized trust infrastructure. In: 49th Annual IEEE/IFIP International Conference on Dependable Systems and Networks (DSN), pp 568-580

Graves A, Mohamed A, Hinton GE (2013) Speech Recognition with Deep Recurrent Neural Networks. CoRR abs/1303.5778, URL http: //arxiv.org/abs/1303.5778 1303.5778

Gray J (1978) Notes on Data Base Operating Systems. In: Operating Systems, An Advanced Course, Springer-Verlag, pp 393 481

Grumberg O, Long DE (1994) Model Checking and Modular Verification. ACM Trans Program Lang Syst 16(3):843-871

Gupta S, Sadoghi M (2018) EasyCommit: A Non-blocking Two-phase Commit Protocol In: Proceedings of the 21st International Conference on Extending Database Technology, Open Proceedings, EDBT

Gupta S, Sadoghi M (2020) Efficient and nonblocking agreement protocols. Distributed Parallel Databases 38(2):287-333, DOI 10. 1007/s10619-019-07267-w

Gupta S, Hellings J, Rahnama S, Sadoghi M (2019a) An in-depth look of BFT consensus in blockchain: Challenges and opportunities. In: Proceedings of the 20th International Middleware Conference Tutorials, ACM, pp 6-10, DOI 10.1145/3366625. 3369437

Gupta S, Hellings J, Sadoghi M (2019b) Brief announcement: Revisiting consensus protocols through wait-free parallelization. In: 33rd International Symposium on Distributed Computing (DISC 2019), vol 146, pp 44:1-44:3, DOI 10.4230/LIPIcs.DISC. 2019.44

Gupta S, Hellings J, Rahnama S, Sadoghi M (2020a) Building high throughput permissioned blockchain fabrics: Challenges and opportunities. Proc VLDB Endow 13(12)

Gupta S, Rahnama S, Hellings J, Sadoghi M (2020b) Resilientdb: Global scale resilient blockchain fabric. PVLDB 13(6):868-883

Gupta S, Rahnama S, Sadoghi M (2020c) Permissioned blockchain through the looking glass: Architectural and implementation lessons learned. To appear in the Proceedings of 40th International Conference on Distributed Computing Systems (ICDCS)
Gupta S, Hellings J, Rahnama S, Sadoghi M (2021a) Proof-of-Execution: Reaching consensus through fault-tolerant speculation. In: Proceedings of the 24th International Conference on Extending Database Technology

Gupta S, Hellings J, Sadoghi M (2021b) RCC: Resilient Concurrent Consensus for HighThroughput Secure Transaction Processing. In: 37th IEEE International Conference on Data Engineering (ICDE), URL http: //arxiv.org/abs/1911.00837 1911.00837

Jakobsson M, Juels A (1999) Proofs of Work and Bread Pudding Protocols. In: Proceedings of the IFIP TC6/TC11 Joint Working Conference on Secure Information Networks: Communications and Multimedia Security, Kluwer, B.V., CMS '99, pp 258272

Katz J, Lindell Y (2007) Introduction to Modern Cryptography. Chapman \& Hall/CRC

Keller RM (1976) Formal verification of parallel programs. Commun ACM 19(7):371384

King S, Nadal S (2012) PPCoin: Peer-to-Peer Crypto-Currency with Proof-of-Stake

Kotla R, Alvisi L, Dahlin M, Clement A, Wong E (2007) Zyzzyva: Speculative Byzantine Fault Tolerance. In: Proceedings of Twentyfirst ACM SIGOPS Symposium on Operating Systems Principles, ACM, New York, NY, USA, SOSP '07, pp 45-58, DOI 10. 1145/1294261.1294267

Krizhevsky A, Sutskever I, Hinton GE (2012) Imagenet classification with deep convolutional neural networks. In: Proceedings of the 25th International Conference on Neural Information Processing Systems - Volume 1, Curran Associates Inc., NIPS'12, pp 10971105

Kumar R, Myreen MO, Norrish M, Owens S (2014) CakeML: A Verified Implementation of ML. In: Proceedings of the 41st ACM SIGPLAN-SIGACT Symposium on Principles of Programming Languages, ACM, New York, NY, USA, POPL '14, pp 179191

Lamport L (1998) The Part-time Parliament. ACM Trans Comput Syst 16(2):133-169

Leroy X (2009) A Formally Verified Compiler Back-end. J Autom Reason 43(4):363-446

Libra (2019) An introduction to libra. Libra Association Members 
Menezes AJ, Vanstone SA, Oorschot PCV (1996) Handbook of Applied Cryptography, 1st edn. CRC Press, Inc.

Nawab F (2018) Geo-Scale Transaction Processing, Springer International Publishing, pp 1-7. DOI 10.1007/978-3-319-63962-8_ 180-1, URL https://doi.org/10. 1007/978-3-319-63962-8_180-1

Oki BM, Liskov BH (1988) Viewstamped Replication: A New Primary Copy Method to Support Highly-Available Distributed Systems. In: Proceedings of the Seventh Annual ACM Symposium on Principles of Distributed Computing, ACM, New York, NY, USA, PODC' '88, pp 8-17

Parity Technologies (2018) Parity Ethereum Blockchain. URL https: //www.parity.io/

Park S, Kwon A, Fuchsbauer G, Gaži P, Alwen J, Pietrzak K (2015) SpaceMint: A Cryptocurrency Based on Proofs of Space. https://eprint.iacr.org/ 2015/528

Pilkington M (2015) Blockchain Technology: Principles and Applications. In: Research Handbook on Digital Transformations, SSRN

Qadah T, Gupta S, Sadoghi M (2020) Q-store: Distributed, multi-partition transactions via queue-oriented execution and communication. In: Proceedings of the 23nd International Conference on Extending Database Technology, EDBT, 2020, OpenProceedings.org, pp 73-84, DOI 10.5441/002/edbt. 2020.08

Qadah TM, Sadoghi M (2018) QueCC: A queue-oriented, control-free concurrency architecture. In: Proceedings of the 19th International Middleware Conference, ACM, Middleware, pp 13-25, DOI 10.1145/ 3274808.3274810

Rahnama S, Gupta S, Qadah TM, Hellings J, Sadoghi M (2020) Scalable, resilient, and configurable permissioned blockchain fabric. Proc VLDB Endow 13(12)

Rosenfeld M (2014) Analysis of hashrate-based double spending. CoRR abs/1402.2009, URL http://arxiv.org/abs/ $1402.2009,1402.2009$

Sadoghi M (2017) Expodb: An exploratory data science platform. In: Proceedings of the Eighth Biennial Conference on Innovative Data Systems Research, CIDR
Sadoghi M, Blanas S (2019) Transaction Processing on Modern Hardware. Synthesis Lectures on Data Management, Morgan \& Claypool, DOI 10.2200/ S00896ED1V01Y201901DTM058

Satoshi N (2008) Bitcoin: A peer-to-peer electronic cash system. URL http:// bitcoin.org/bitcoin.pdf

Schwartz D, Youngs N, Britto A (2014) The Ripple Protocol Consensus Algorithm. URL https://www.ripple.com/

Singh A, Das T, Maniatis P, Druschel P, Roscoe T (2008) BFT Protocols under Fire. In: NSDI

Skeen D (1982) A Quorum-Based Commit Protocol. Tech. rep.

Steen Mv, Tanenbaum AS (2017) Distributed Systems

TPP Council (2010) TPC Benchmark C (Revision 5.11)

Wilcox JR, Woos D, Panchekha P, Tatlock Z, Wang X, Ernst MD, Anderson T (2015) Verdi: A Framework for Implementing and Formally Verifying Distributed Systems. In: Proceedings of the 36th ACM SIGPLAN Conference on Programming Language Design and Implementation, ACM, New York, NY, USA, PLDI '15, pp 357-368

Wood G (2015) Ethereum: A secure decentralised generalised transaction ledger. URL http://gavwood.com/paper.pdf

Yin M, Malkhi D, Reiter MK, Gueta GG, Abraham I (2019) HotStuff: BFT consensus with linearity and responsiveness. In: Proceedings of the 2019 ACM Symposium on Principles of Distributed Computing, ACM, PODC, pp 347-356, DOI 10.1145/3293611. 3331591

Zhao W, Chellappa R, Phillips PJ, Rosenfeld A (2003) Face Recognition: A Literature Survey. ACM Comput Surv 35(4):399-458, DOI $10.1145 / 954339.954342$ 